\title{
IOX1, a JMJD2A inhibitor, suppresses the proliferation and migration of vascular smooth muscle cells induced by angiotensin II by regulating the expression of cell cycle-related proteins
}

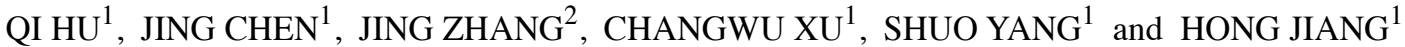 \\ ${ }^{1}$ Department of Cardiology, Renmin Hospital of Wuhan University, Cardiovascular Research Institute of Wuhan University, \\ Wuhan, Hubei 430060; ${ }^{2}$ Department of Cardiology, The First College of Clinical Medical Sciences, \\ China Three Gorges University, Yichang, Hubei 443000, P.R. China
}

Received February 25, 2015; Accepted October 1, 2015

DOI: $10.3892 /$ ijmm.2015.2393

\begin{abstract}
The epigenetic modification of vascular smooth muscle cell (VSMC) phenotypic switching, proliferation, migration, apoptosis and extracellular matrix synthesis is known to occur in atherosclerosis. The aim of the present study was to investigate the effects of IOX1, a Jumonji domaincontaining 2A (JMJD2A) inhibitor, on regulation of the cell cycle in angiotensin II (Ang II)-stimulated VSMCs and to elucidate the possible mechanisms involved. The proliferation and migration of the Ang II-stimulated VSMCs in the presence or absence of IOX1 were evaluated in vitro. Flow cytometric analysis was used to determine the effects of IOX1 on cell cycle progression. RT-qPCR and western blot analysis were carried out to measure the expression levels of cell cycle-related genes. The trimethylation of histone $\mathrm{H} 3$ lysine 9 (H3K9me3) at the promoters of these genes was detected by chromatin immunoprecipitation (ChIP) assay. We confirmed that the JMJD2A levels were increased, whereas the H3K9me3 levels were decreased in the Ang II-stimulated VSMCs. The inhibition of JMJD2A by IOX1 suppressed the Ang II-induced cell proliferation, migration and cell cycle progression by inhibiting cyclin D1 expression and increasing p21 expression. The underlying mechanisms were related to the restoration of the $\mathrm{H} 3 \mathrm{~K} 9 \mathrm{me} 3$ levels at the promoters of these genes. In conclusion, the findings of our study indicate that IOX1 exerts its anti-proliferative and anti-migratory effects by regulating the expression of the cell cycle-related proteins, cyclin D1 and p21.
\end{abstract}

Correspondence to: Professor Hong Jiang or Dr Jing Chen, Department of Cardiology, Renmin Hospital of Wuhan University, Cardiovascular Research Institute of Wuhan University, 238 Jiefang Road, Wuhan, Hubei 430060, P.R. China

E-mail: pyh_hongj@163.com

E-mail: chenjing821111@163.com

Key words: Jumonji domain-containing 2A, histone methylation, vascular smooth muscle cells, angiotensin II, cell cycle, cyclin D1, p21

\section{Introduction}

Atherosclerosis, which poses a serious threat to human health, is an important public health concern. The development of atherosclerosis involves a complex pathogenic process which includes endothelial dysfunction, the entry and retention of lipids, leukocyte adhesion, the migration and proliferation of vascular smooth muscle cells (VSMCs) and the increased synthesis of the extracellular matrix (1). Disorders in the functions of VSMCs play a vital role in vascular proliferative diseases, including atherosclerosis and restenosis following coronary angioplasty (2). Atherogenic stimuli, including cytokines, lipids, shear stress and reactive oxygen species, provoke VSMCs migrating from the media to the intima, to switch from a quiescent 'contractile' state to a 'synthetic' phenotypic state and to enter the cell cycle. Cell cycle progression is regulated by a range of regulatory proteins, including cyclins, cyclin-dependent kinases (CDKs) and CDK inhibitors (CDKIs). The cyclin D/ CDK4/6 complex is active in the early G1 phase of the cell cycle, regulating G1 phase progression, whereas cyclin E/CDK2 or cyclin A/CDK2 are essential for the G1/S phase transition. Cell cycle progression is blocked by CDKIs, such as p27 and p21 (3).

Angiotensin II (Ang II), the main member of the reninangiotensin-aldosterone system (RAAS), is an important regulator of the cardiovascular system (4). Ang II induces the growth and migration of smooth muscle cells, which contributes to the progression and development of cardiovascular disease through the activation of the mitogen-activated protein kinase (MAPK) signaling cascade, including extracellular signal-regulated kinase (ERK1/2), JNK and p38 MAPK (5). MAPK activation increases $c$-fos and $c$-jun gene expression and promotes the translocation of activator protein-1 (AP-1) to the nucleus, where it binds to the promoter of various target genes, such as cyclin D1 (6).

Jumonji domain-containing 2A (JMJD2A), also known as KDM4A, belongs to the Jumonji C (JmjC) domain-containing family of JMJD2 proteins. It is a lysine trimethyl-specific histone demethylase that catalyses the demethylation of trimethylated histone H3 lysine 9 (H3K9me3) and H3K36 (H3K36me3) (7). 
Through JMJD2A activity, H3K9me3 demethylation contributes to an open chromatin state and is connected with the transcriptional activation of promoter regions (8).

In the present study, we investigated the role of JMJD2A in the dysfunction of VSMCs following stimulation with Ang II. In addition, we aimed to investigate whether IOX1, a known JMJD2A inhibitor, can suppress the proliferation and migration of VSMCs induced by Ang II, as well as the underlying mechanisms.

\section{Materials and methods}

JMJD2A inhibitor. The JMJD2A inhibitor, IOX1 [5-carboxy8-hydroxyquinoline (5-carboxy-8-HQ)], was dissolved in dimethyl sulfoxide (DMSO) (both from Sigma, St. Louis, MO, USA) to yield a $250 \mu \mathrm{M}$ stock solution and was subsequently diluted in Dulbecco's modified Eagle's medium (DMEM; HyClone, Logan, UT, USA) to the required concentration.

Primary culture of VSMCs. As previously described (9), VSMCs were isolated from the thoracic aortas of male Sprague-Dawley rats (weighing, 150-180 g). All animals used in our study were purchased from the Animal Center of Renmin Hospital of Wuhan University. The experimental procedures and animal care procedures were conducted in accordance with the Institutional Animal Care and Use Committee of Wuhan University. Briefly, rat thoracic aortas were harvested following euthanasia. After scraping off the adventitia and endothelium, thoracic aortas were cut into sections of $2 \times 2 \mathrm{~mm}$. The primary VSMCs were obtained using the tissue explants adherent method and cultured in DMEM (HyClone) supplemented with $10 \%$ fetal bovine serum (FBS; Gibco, Grand Island, NY, USA) and $1 \%$ penicillin/streptomycin (PS; Beyotime Institute of Biotechnology, Bejing, China) in an atmosphere of $5 \% \mathrm{CO}_{2}$, at $37^{\circ} \mathrm{C}$. The purity of the VMSCs was assessed by immunostaining with anti-smooth muscle $\alpha$-actin antibody. VSMCs between the third and fifth passages were used for the experiments.

Cell proliferation assay. The cell proliferation assay was performed using a Cell Counting Kit-8 (CCK8; Dojindo Molecular Technologies, Inc., Kumamoto, Japan). Briefly, approximately 8,000 VSMCs were seeded in 96-well plates in $200 \mu \mathrm{l}$ of culture medium. Following synchronization with DMEM containing $0.1 \%$ FBS for $24 \mathrm{~h}$, the VSMCs were pretreated with IOX1 at various concentrations $(0,50,100$ and $200 \mu \mathrm{M}$ ) for $2 \mathrm{~h}$, and then stimulated with $1 \mu \mathrm{M}$ Ang II (Sigma) for $24 \mathrm{~h}$. CCK8 solution $(20 \mu \mathrm{l})$ was then added and the OD value was measured at $450 \mathrm{~nm}$ using a microplate spectrophotometer (Infinite M200; Tecan Group Ltd., Männedorf, Switzerland).

Cell migration assay. A Transwell chamber assay (Corning Inc., Corning, NY, USA) was performed to evaluate the migration activity of the VMSCs. Synchronized $\left(10^{5}\right)$ VSMCs were seeded into the upper chamber in $200 \mu \mathrm{l}$ of serum-free medium in the presence or absence of IOX1. Following inhibitor precondition for $2 \mathrm{~h}, 600 \mu \mathrm{l}$ of DMEM supplemented with $1 \mu \mathrm{M}$ Ang II were added to the lower chamber followed by incubation for $8 \mathrm{~h}$. Cells remaining on the upper side of the membrane were gently wiped with a cotton swab. The migrated cells were fixed with methanol and stained with $0.5 \%$ crystal violet. The number of migrated cells was counted in 5 random fields under a microscope (Olympus Corporation, Tokyo, Japan) at a x100 magnification.

Cell cycle analysis. Cell-cycle analysis was carried out by flow cytometry. The VSMCs were cultured in 6-cm culture plates (Corning Inc.) at a density of $5 \times 10^{5}$ cells/plate. At $50 \%$ cell density, the VSMCs were synchronized for $24 \mathrm{~h}$. Then VSMCs were then pre-treated with IOX1 $(200 \mu \mathrm{M})$, followed by treatment with Ang II treatment for $24 \mathrm{~h}$. The VMSCs were then trypsinized, harvested and washed twice with cold phosphate-buffered saline (PBS), and fixed with $70 \%$ cold ethanol and stored at $4^{\circ} \mathrm{C}$ overnight. After staining with propidium iodide (PI solution: $50 \mathrm{mg} / \mathrm{ml} \mathrm{PI,} 100 \mathrm{mg} / \mathrm{ml}$ RNase A) at $37^{\circ} \mathrm{C}$ for $30 \mathrm{~min}$, the cell cycle distribution was determined by flow cytometry (BD FACSCalibur; Becton, Dickinson and Company, Franklin Lakes, NJ, USA). The percentage of cells in the G0/G1, S and G2-M phase of the cell cycle was determined using ModFitLT cell cycle analysis software (Verity Software House Inc., Topsham, ME, USA).

Western blot analysis. The synchronized VSMCs were pretreated with IOX1 $(200 \mu \mathrm{M})$ for $2 \mathrm{~h}$ and then stimulated with Ang II for 0, 3, 6, 12 and $24 \mathrm{~h}$ and lysed in RIPA lysis buffer (Beyotime Institute of Biotechnology) containing $1 \mathrm{mM}$ PMSF and $10 \mathrm{mM}$ phosphatase inhibitor (Roche, Mannheim, Germany) on ice for $15 \mathrm{~min}$, and centrifuged for $5 \mathrm{~min}$ at $12,000 \mathrm{x} \mathrm{g}$ at $4^{\circ} \mathrm{C}$. Total protein was quantified using a BCA protein assay kit (Beyotime Institute of Biotechnology) according to the manufacturer's instructions. Equal amounts of protein $(35 \mu \mathrm{g})$ were separated by sodium dodecyl sulfate-polyacrylamide gel electrophoresis (SDS-PAGE) and transferred onto polyvinylidene difluoride (PVDF) membranes. The PVDF membranes were blocked and incubated with antibodies against JMJD2A (SAB3500095; Sigma), H3K9me3 (ab8898; Abcam, Cambridge, MA, USA), cyclin D1 (2978T), cyclin E (4136S), p21 (2947T), p27 (3686T; all from Cell Signaling Technology, Inc., Danvers, MA, USA) and GAPDH (5632-1; Epitomics, Burlingame, CA, USA) overnight at $4^{\circ} \mathrm{C}$. The washed membranes were incubated with horseradish peroxidase (HRP)-conjugated secondary antibodies (7074; Cell Signaling Technology, Inc.) for $2 \mathrm{~h}$ at room temperature, and subsequently detected using ECL reagent (Pierce, Rockford, IL, USA). The results of the western blot analysis were quantified using Quantity One software.

Reverse transcription-quantitative polymerase chain reaction $(R T-q P C R)$. The mRNA expression levels of cyclin D1 and $\mathrm{p} 21$ were measured by RT-qPCR. The synchronized VSMCs were pre-treated with IOX1 $(200 \mu \mathrm{M})$ for $2 \mathrm{~h}$ and then stimulated with Ang II for $12 \mathrm{~h}$. Total RNA was extracted using the PicoPure RNA isolation kit (Applied Biosystems Life Technologies, Carlsbad, CA, USA) according to the manufacturer's instructions and reverse transcribed into cDNA using a First-Strand Synthesis system (Invitrogen, Carlsbad, CA, USA). This was performed using the SYBR system on the ABI Prism 7500 Sequence Detection System (Applied Biosystems Life Technologies, Foster City, CA, USA). Each sample was run and analyzed in triplicate. Data were normalized to GAPDH expression and analyzed using the $2^{-\Delta \Delta \mathrm{Ct}}$ method. The primer sequences used in the present study were as follows: cyclin D1 forward, 5'-TGTTCGTGGCCTCTAAGAT 

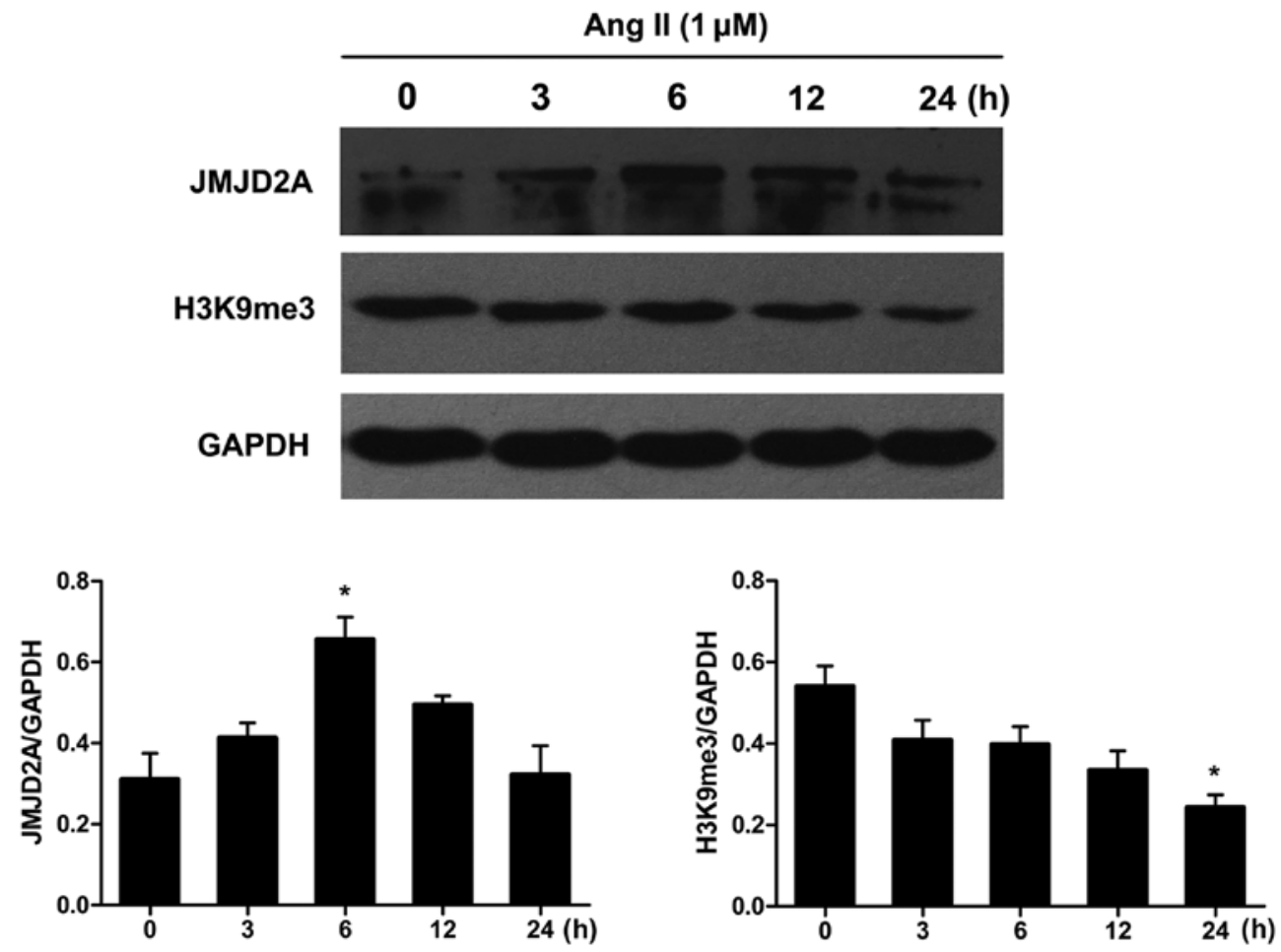

Figure 1. Expression of Jumonji domain-containing 2A (JMJD2A) and H3K9me3 in angiotensin II (Ang II)-stimulated vascular smooth muscle cells (VSMCs). VSMCs were treated with $1 \mu \mathrm{M}$ Ang II for 0-24 h. Total protein levels of JMJD2A and H3K9me3 were measured by western blot analysis and quantified using Quantity One software. Ang II increased the expression of JMJD2A and decreased the expression of H3K9me3. Data are presented as the means \pm SEM of 3 experiments. " $\mathrm{p}<0.05$ vs. $0 \mathrm{~h}$.

GAAG-3' and reverse, 5'-GGAAGTGTTCGATGAAATC GTG-3'; p21 forward, 5'-AGTATGCCGTCGTCTGTTCG-3' and reverse, 5'-GAGTGCAAGACAGCGACAAG-3'; and GAPDH forward, 5'-GACATGCCGCCTGGAGAAAC-3' and reverse, 5'-AGCCCAGGATGCCCTTTAGT-3'.

Chromatin immunoprecipitation (ChIP) assay. ChIP assay was performed using a ChIP assay kit (Pierce) according to the manfacturer's instructions. Briefly, the synchronized VSMCs were pre-treated with IOX1 $(200 \mu \mathrm{M})$ for $2 \mathrm{~h}$ and then stimulated with Ang II for $12 \mathrm{~h}$ and fixed with $1 \%$ formaldehyde, washed and lysed. The cell lysates were sonicated to create chromatin fragments of 400-500 bp in length, diluted in ChIP dilution buffer and subjected to immunoprecipitation with anti-H3K9me3 antibodies (ab8898; Abcam) overnight at $4^{\circ} \mathrm{C}$. The immune complexes were collected, washed and eluted with buffer. Protein-DNA cross-links were reversed overnight at $65^{\circ} \mathrm{C}$ and the DNA was extracted. ChIP-enriched DNA samples were analyzed by RT-qPCR using primers specific for cyclin D1 or p21. Data were analyzed using the $2^{-\Delta \Delta \mathrm{Ct}}$ method and normalized with input samples. The results are expressed as a percentage (\%) of the control values. The primer sequences were follows: cyclin D1 forward, CTCTGCCCGGCTTTGAT CTCT-3' and reverse, 5'-AGGCTGCAGGACT TTGCAACT-3'; and p21 forward, 5'-GTTCAGCCCTGGAACCGAAG-3' and reverse, 5'-GTACCAAACACCCTTCACCTGGTAC-3'.

Statistical analysis. All results were analyzed using either a Student's t-test for between-group comparisons or the one-way ANOVA for multiple comparisons using SPSS 13 software.
Data are presented as the means \pm SEM of 3 or 6 experiments, as indicated in the figure legends. A value of $\mathrm{p}<0.05$ was considered to indicate a statistically significant difference.

\section{Results}

JMJD2A and H3K9me3 protein expression levels in Ang II-stimulated VSMCs. Subconfluent, quiescent VSMCs were exposed to Ang II for 0, 3, 6, 12 and $24 \mathrm{~h}$. Western blot analysis was performed to assess the total protein levels of JMJD2A and its primary target, H3K9me3, in Ang II-stimulated VSMCs. The time course of JMJD2A and H3K9me3 protein expression is shown in Fig. 1. Ang II induced a slight increase in the JMJD2A protein levels at an early time point $(3 \mathrm{~h})$. The levels peaked at around $6 \mathrm{~h}$ and were maintained at these levels until the 12-h time point. Conversely, the protein expression of $\mathrm{H} 3 \mathrm{~K} 9 \mathrm{me} 3$ decreased in a time-dependent manner in the Ang II-stimulated VSMCs.

IOXI suppresses the proliferation and migration of VMSCs induced by Ang II. Since the expression of JMJD2A was upregulated in the Ang II-stimulated VSMCs, we intended to block JMJD2A with the specific inhibitor, IOX1, and to examine the effect of JMJD2A inhibition on Ang II-stimulated VSMCs. A significant increse in proliferation was observed in the subconfluent, quiescent VSMCs following stimulation with Ang II in the absence of serum (Fig. 2). The VSMCs pre-treated with IOX1 $(0,50,100$ and $200 \mu \mathrm{M})$ exhibited a decrease in proliferation in a dose-dependent manner. The most prominent suppressive effect on VSMC proliferation was 


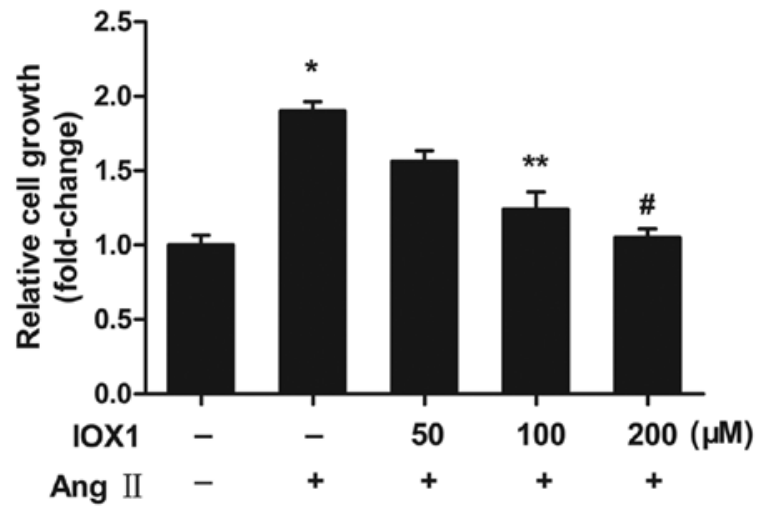

Figure 2. IOX1 inhibits the proliferation of vascular smooth muscle cells (VSMCs) stimulated with angiotensin II (Ang II). VSMCs were pretreated with IOX1 for $2 \mathrm{~h}$ at concentrations varying from 0 to $200 \mu \mathrm{M}$ and stimulated with $1 \mu \mathrm{M}$ Ang II for a further $24 \mathrm{~h}$. IOX1 inhibited the Ang II-induced proliferation in a concentration-dependent manner. Data are presented as the means \pm SEM of 6 experiments. ${ }^{*} p<0.05$ vs. control group (no treatment); ${ }^{* *} \mathrm{p}<0.05$ vs. cells treated with Ang II alone; ${ }^{\#} \mathrm{p}<0.05$ vs. cells treated with Ang II alone.

observed following treatment with IOX1 at $200 \mu \mathrm{M}$ (decreased in proliferation of $44.73 \%$ ). We then wished to examine whether IOX1 suppresses the migration of Ang II-stimulated VSMCs. Pretreated VSMCs pre-treated with $200 \mu \mathrm{M}$ IOX1 were stimulated with Ang II. The enhanced migration of the Ang II-stimulated VSMCs was indeed suppressed by pretreatment with IOX1 (Fig. 3). These data indicated that IOX1 inhibited the proliferation and migration of VSMCs stimulated with Ang II.
IOXI blocks the cell cycle progression of VSMCs stimulated with Ang II. We then examined the effects of IOX1 on the cell cycle of VSMCs. Following absolute synchronization, the VSMCs were stimulated with Ang II for $24 \mathrm{~h}$ in the presence or absence of IOX1. Cell cycle analysis using flow cytometry revealed that there was a marked increase in the percentage of Ang II-stimulated cells in the S phase compared with the quiescent (unstimulated) cells. This increase in the $\mathrm{S}$ phase cell population was decreased by IOX1 treatment (Fig. 4). The percentage of cells in the G0/G1 phase were increased in the cells pre-treated with IOX1 (Fig. 4), indicating that IOX1 significantly inhibited the proliferation of the VSMCs by slowing down the progression of the cell cycle from the G0/G1 to the $\mathrm{S}$ phase.

Effect of IOXI on the expression of cell cycle-related genes. Considering that IOX1 suppressed the proliferation of VSMCs and blocked cell cycle progression, particularly at the G1/S phase transition, we measured the expression levels of various cell cycle-related proteins in the Ang II-stimulated VSMCs in the presence or absence of IOX1 $(50,100$ and $200 \mu \mathrm{M})$. Stimulation with Ang II increased the expression of cyclin D1 and cyclin E (Fig. 5). This increase in cyclin D1 expression was attenuated by IOX1 in a concentration-dependent manner. However, IOX1 had a marginal effect on cyclin E expression. In addition, we measured the protein expression levels of the CDKIs, p21 and p27. The levels of p27 were not influenced by Ang II, whereas the p21 levels were decreased in the Ang II-stimulated VSMCs. When the cells were pre-treated with to IOX1, p21 expression was upregulated in the Ang II-stimulated VSMCs. To determine whether the changes in the protein expression
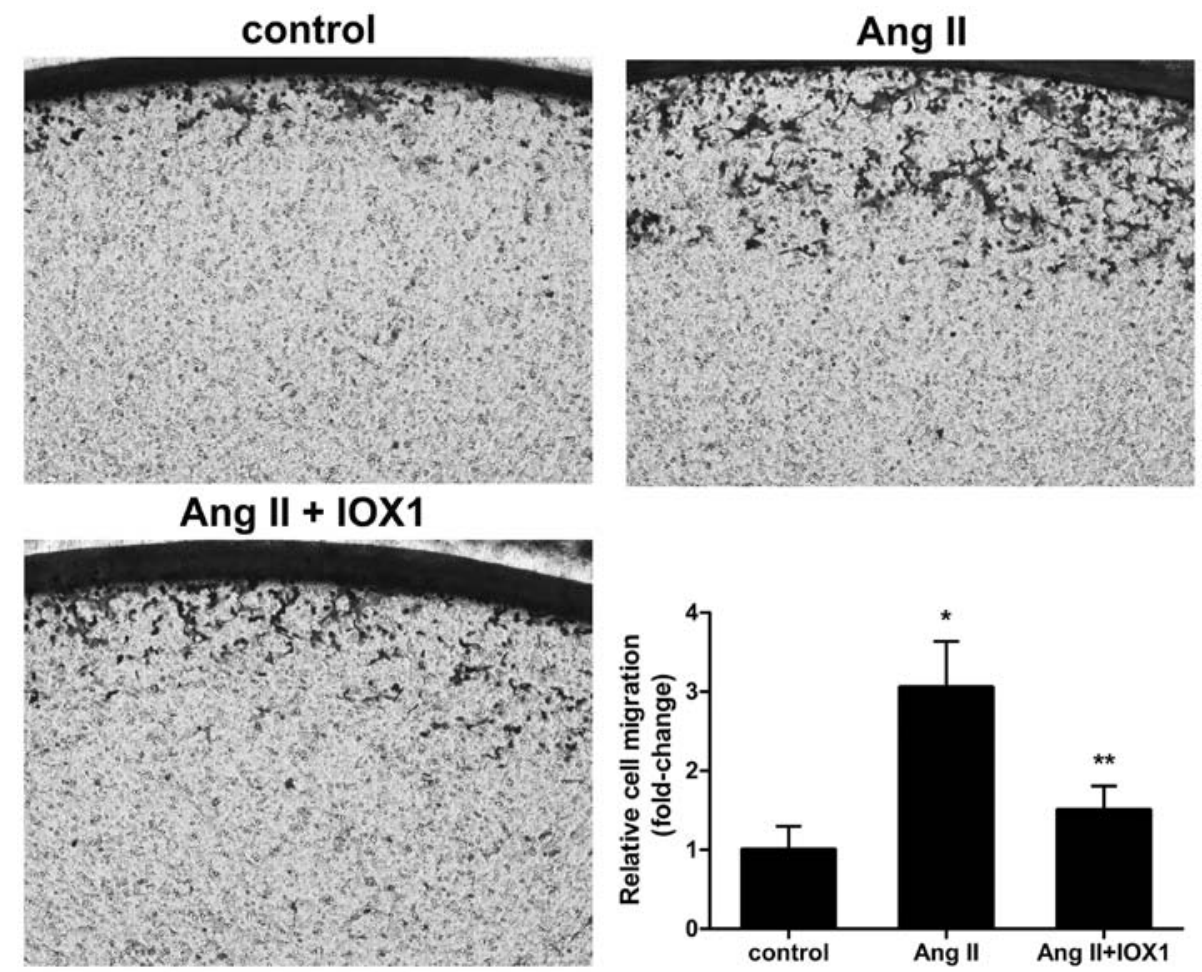

Figure 3. IOX1 inhibits the migration of vascular smooth muscle cells (VSMCs) stimulated with angiotensin II (Ang II). VSMCs were pre-treated with $200 \mu \mathrm{M}$ IOX1 and then stimulated with or without $1 \mu \mathrm{M}$ Ang II for $8 \mathrm{~h}$. Cellular migration was detected by Transwell assay. Data are presented as the means \pm SEM of 3 experiments. ${ }^{*}<<0.05$ vs. control group; ${ }^{* *} \mathrm{p}<0.05$ vs. cells treated with Ang II alone. Magnification, $\mathrm{x} 100$. 

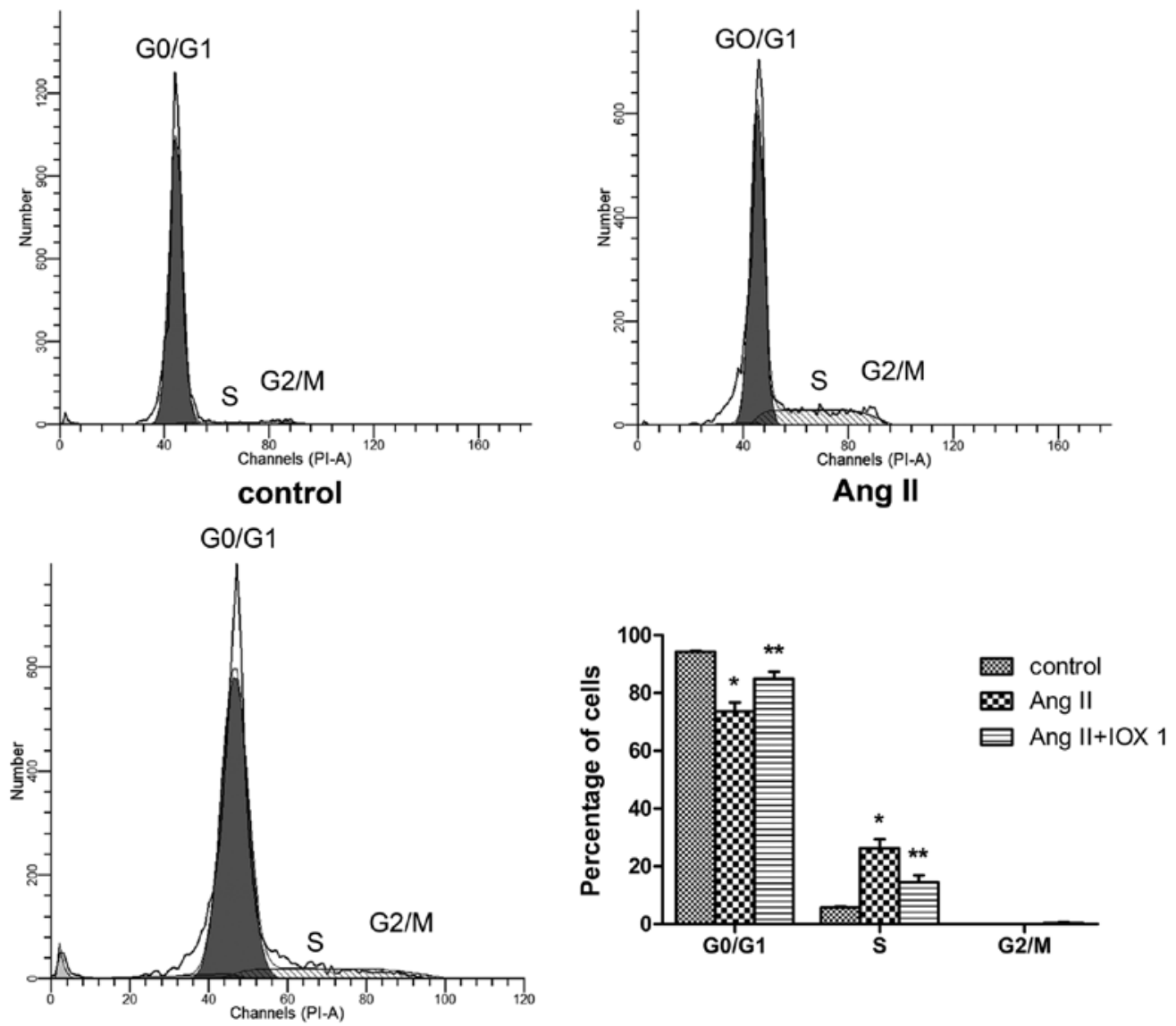

Ang $11+10 X 1$

Figure 4. IOX1 blocks the cell cycle progression of angiotensin II (Ang II)-stimulated vascular smooth muscle cells (VSMCs). Synchronized VSMCs were stimulated with $1 \mu \mathrm{M}$ Ang II for $24 \mathrm{~h}$ in the presence or in the absence of $200 \mu \mathrm{M}$ IOX1. Analysis of the cell cycle was performed by flow cytometry. IOX1 blocked the Ang II-induced cell cycle progression. Data are presented as the means \pm SEM of 3 experiments. ${ }^{*} p<0.05$ vs. control group; ${ }^{* *} p<0.05$ vs. cells treated with Ang II alone.

levels of cyclin D1 and p21 are regulated by IOX1 at the transcriptional level, RT-qPCR was performed to measure their mRNA expression levels. The mRNA expression levels were similar to the protein levels. IOX1 decreased cyclin D1 mRNA expression and upregulated p21 mRNA expression (Fig. 6). These data suggested that IOX1 inhibited the Ang II-induced VSMC proliferation through selective regulation of the cell cycle-related proteins, cyclin D1 and p21.

IOX1 mediates cyclin D1 and p21 expression by regaining H3K9me3 at the promoters of these genes. To determine whether IOX1 epigenetically mediates the expression of cyclin D1 and p21 in Ang II-stimulated VSMCs, we first examined the global changes in $\mathrm{H} 3 \mathrm{~K} 9 \mathrm{me}$, the main substrate of JMJD2A. IOX1 enhanced the total protein levels of H3K9me3 which were reduced in the Ang II-stimulated VSMCs (Fig. 7A). To investigate the mechanisms through which IOX1 regulates cyclin D1 and p21 expression, ChIP assays were performed using antiH3K9me3 to examine the effects of IOX1 on the cyclin D1 and p21 promoters in the Ang II-stimulated VSMCs. The results revealed that the levels of $\mathrm{H} 3 \mathrm{~K} 9 \mathrm{me} 3$ in the cyclin D1 and p21 promoter regions were decreased in the Ang II-stimulated cells compared to to the untreated control cells. Pre-treatment with $200 \mu \mathrm{M}$ of IOX1 increased the levels of H3K9me3 in the promoters of these genes (Fig. 7B).

\section{Discussion}

The abnormal growth of VSMCs has been shown to contribute to the development of occlusive vascular diseases, such as atherosclerosis and restenosis following coronary angioplasty (2). It is important to elucidate the underlying mechanisms responsible for the dysfunction of VSMCs in a pathogenic environment. In this study, we found that the levels of JMJD2A, a newly recognized histone demethylase, were increased in the Ang II-stimulated VSMCs, and this was accompanied by suppressed global levels of H3K9me3. Thus, we utilized IOX1, a selective inhibitor of the Jumonji protein subtypes, to examine the effects of the inhibition of JMJD2A on VSMC proliferation and migration. We used Ang II as a proliferative agent which has been demonstrated to be a critical mediator of cardiovascular disease progression (4). IOX1 suppressed the proliferation and migration of VSMCs induced by Ang II, exerting anti-proliferative and anti-migratory effects by regulating the expression of cell cycle-related proteins, cyclin D1 and p21. expression. ChIP assays demonstrated that IOX1 altered the expression of the cell cycle proteins by modifying the level of $\mathrm{H} 3 \mathrm{~K} 9 \mathrm{me} 3$ at the promoters of these genes.

JMJD2A, also known as KDM4A, belongs to the JmjC domain-containing family of JMJD2 proteins. It is a lysine trimethyl-specific histone demethylase that catalyses the 

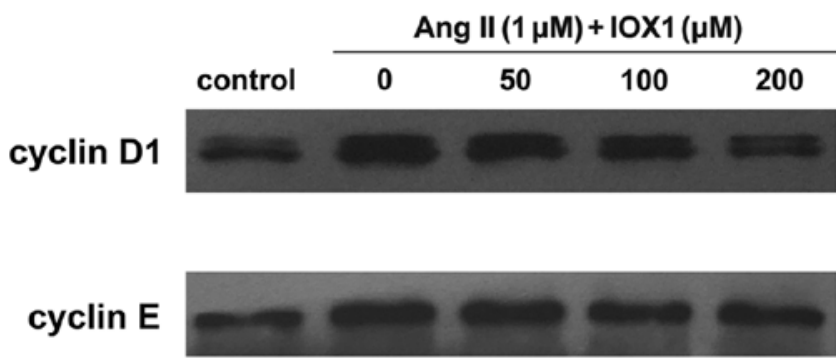

p21

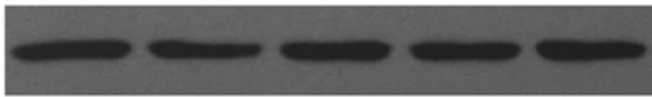

p27

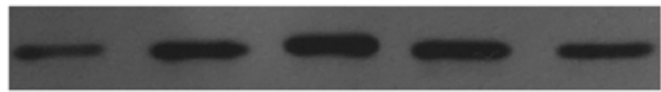

GAPDH
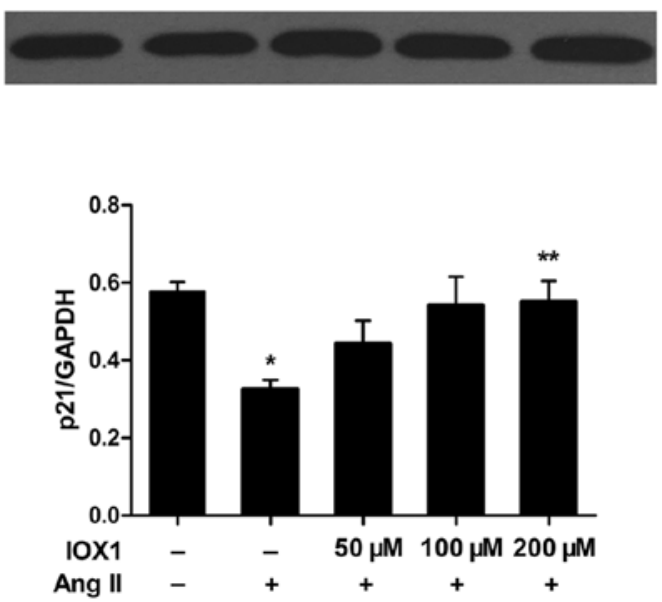
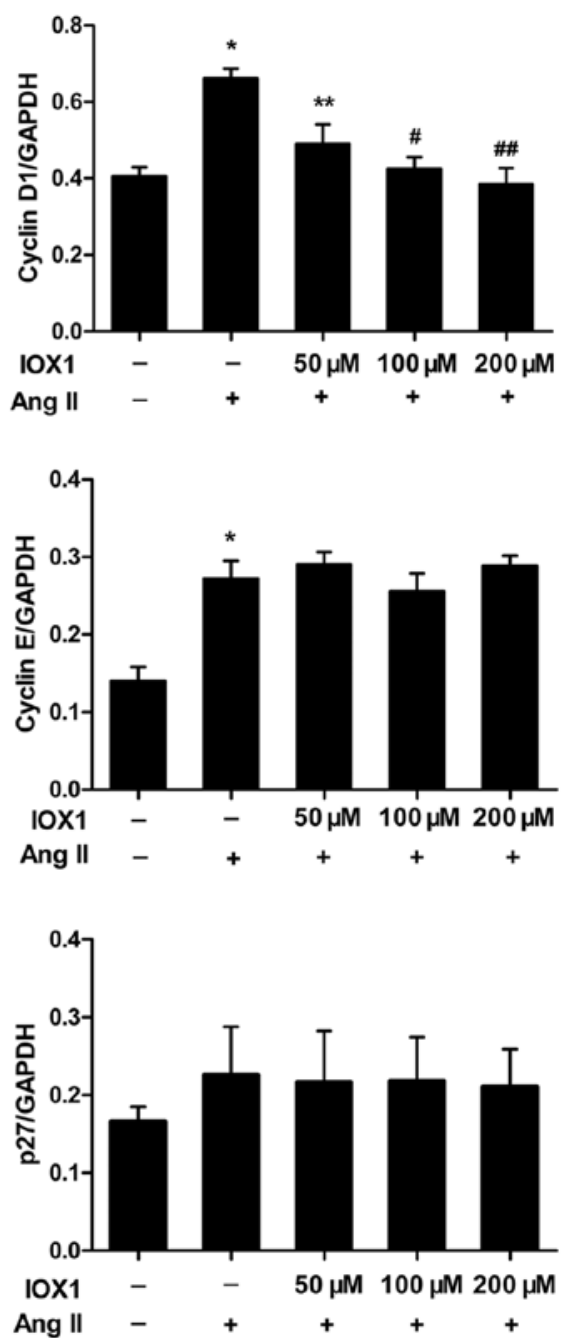

Figure 5. Effect of IOX1 on the expression of cell cycle-related proteins. Vascular smooth muscle cells (VSMCs) were treated with IOX1 for $2 \mathrm{~h}$, followed by stimulation with $1 \mu \mathrm{M}$ angiotensin II (Ang II) for $12 \mathrm{~h}$ and the expression of cell cycle-related proteins was the measured by western blot analysis. IOX1 reduced cyclin D1 and increased p21 levels. It had no effect on cyclin E and p27 expression. Data are presented as the means \pm SEM of 3 experiments. "p $<0.05$ vs. control group; ${ }^{* *} \mathrm{p}<0.05$ vs. cells treated with Ang II alone; ${ }^{\#} \mathrm{p}<0.05$ vs. cells treated with Ang II alone; ${ }^{* \#} \mathrm{p}<0.05$ vs. cells treated with Ang II alone.
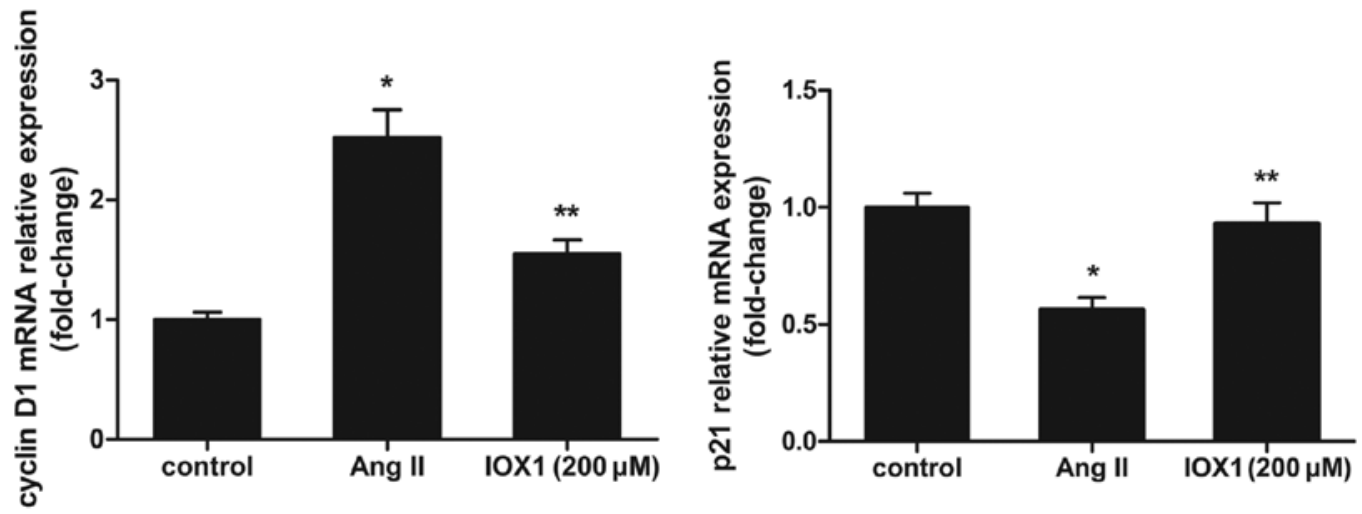

Figure 6. Effect of IOX1 on the mRNA expression of cyclin D1 and p21. Vascular smooth muscle cells (VSMCs) were stimulated with $1 \mu \mathrm{M}$ angiotensin II (Ang II) in the presence and absence of $200 \mu \mathrm{M}$ IOX1. The mRNA expression levels of cyclin D1 and p21 were measured by RT-qPCR. IOX1 reduced cyclin D1 levels and induced the expression of p21. Data are presented as the means \pm SEM of 3 experiments. " $p<0.05$ vs. control group; ${ }^{* *}$ p $<0.05$ vs. cells treated with Ang II alone.

demethylation of H3K9me3 and H3K36me3 (7). It has been suggested that there is an alteration in the JMJD2A expression pattern in several types of cancer (10-15). JMJD2A is involved in the epigenetic regulation of tumor-related gene expression 

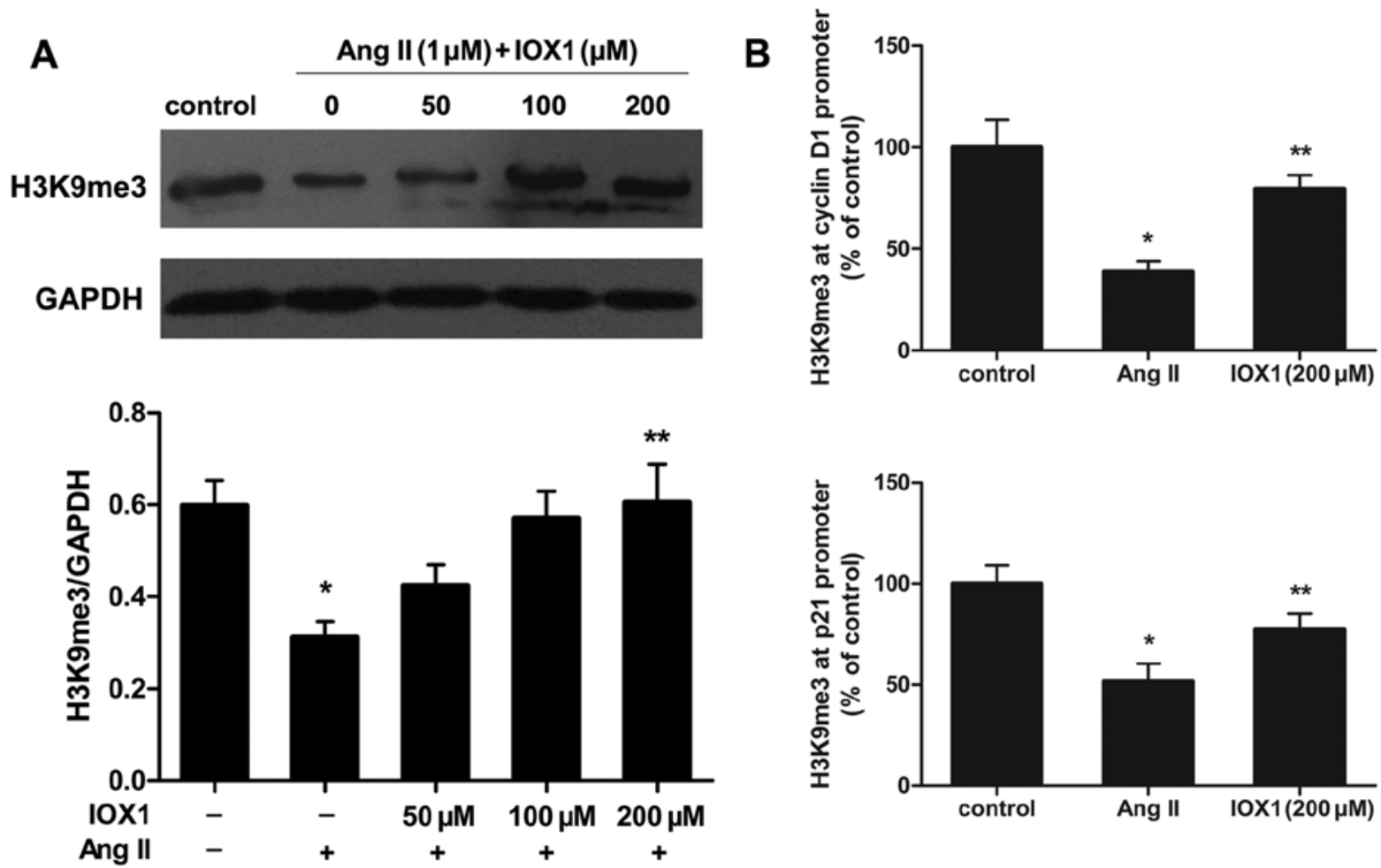

Figure 7. Effect of IOX1 on H3K9me3. Vascular smooth muscle cells (VSMCs) were treated with angiotensin II (Ang II) in the presence and absence of $200 \mu \mathrm{M}$ IOX1. The global H3K9me3 and H3K9me3 expression levels at the promoters were measured by (A) western blot analysis and (B) chromatin immunoprecipitation (ChIP) assay. IOX1 restored H3K9me3 levels. Data are presented as the means \pm SEM of 3 experiments. " $p<0.05$ vs. control group; ${ }^{* *}$ p $<0.05$ vs. cells treated with Ang II alone.

which depends on the demethylation of $\mathrm{H} 3 \mathrm{~K} 9 \mathrm{me} 3$ or the interaction with the tumor suppressor, retinoblastoma protein $(\mathrm{Rb})$, histone deacetylases (HDACs), and the nuclear receptor co-repressor $(\mathrm{N}-\mathrm{CoR})(16,17)$. Previous studies have identified some H3K9me3-related histone methyltransferases and demethylases, such as Suv39h1, G9a and JMJD1A, which participate in the epigenetic regulation of dysfunctional VSMCs in atherosclerosis (18-20). To the best of our knowledge, there is no study available to date on the connection between atherosclerosis and JMJD2A. In the present study, we demonstrated that Ang II induced total JMJD2A expression, and this was accompanied by a suppressed global expression of $\mathrm{H} 3 \mathrm{~K} 9 \mathrm{me} 3$. Based on the above results, we postulated that JMJD2A and its substrate, H3K9me3, participate in the dysfunction of Ang II-stimulated VSMCs.

The cell cycle is a complex process that involves multiple regulatory proteins, including cyclins, $\mathrm{CDKs}$ and CDKIs. In line with previous studies, in the present study, we demonstrated that, following stimulation with Ang II, the expression of cyclin D1 and cyclin E increased markedly $(21,22)$. The Ang II-induced cyclin D1 expression was significantly inhibited by IOX1, whereas the expression of cyclin E was not affected. In addition, treatment of the VSMCs with IOX1 increased p21 expression that had been decreased by stimulation with Ang II. These results suggested that IOX1 blocks cell cycle progression by regulating cyclin D1 and p21 expression. The abnormal expression of cyclin D1 is a hallmark of proliferative diseases, including cancer and atherosclerosis $(23,24)$. Cyclin D1 is regulated transcriptionally and post-transcriptionally (25). In this study, we confirmed that IOX1 inhibited the expression of cyclin D1 by increase $\mathrm{H} 3 \mathrm{~K} 9 \mathrm{me} 3$ expression in the promoter region, reaffirming that $\mathrm{H} 3 \mathrm{~K} 9 \mathrm{me} 3$ is related to heterochromatin formation and transcriptional repression (26). It has been demonstrated that the removal of $\mathrm{H} 3 \mathrm{~K} 9 \mathrm{me} 3$ by JMJD2A is required for the recruitment of AP-1 to chromatin, thereby promoting the positive feedback loop that induces the activation of AP-1 (12). Previous studies have reported that AP-1 transcription factors regulate cyclin D1 gene expression (27). IOX1 may suppress cyclin D1 expression by inhibiting the activation of AP-1. Cyclin D1 is a target gene of estrogen receptor $\alpha(\mathrm{ER} \alpha)(28)$. Berry et al (13) demonstrated that the downregulation of JMJD2A reduced the transcription of a seminal ER $\alpha$ target gene, cyclin D1, which was consistent with our results. Our results, however, seemed to be self-contradictory as IOX1 increased p21 expression. Our observation was in agreement with that of the study by Kim et al, demonstrating that JMJD2A depletion led to increased levels of H3K9me3 at the $\mathrm{p} 21$ promoter and to increased levels of p21 (15).

IOX1, a cell-permeable derivative of 8-hydroxyquinoline, is the most potent broad-spectrum inhibitor of the 2-oxoglutarate (2OG)-dependent JmjC family of histone lysine demethylases (29). It exerts its inhibitory effect on JMJDs by chelating with the $\mathrm{Fe}$ (II) ion to form a bidentate structure positioned at the active site (30). It was demonstrated to inhibit JMJD2A activity in a cell (29). To the best of our knowledge, there is no study available to date on the role of IOX1 in VSMCs. We proved that IOX1 inhibited VSMC proliferation and migration induced by Ang II. IOX1 may also inhibit other JmjC-domain containing histone lysine demethylases. Thus, there may be other potential mechanisms involved and further investigations are warranted. 
In conclusion, in this study, we investigated the inhibitory effect of IOX1 on the proliferation and migration of VMSCs induced by Ang II. We found that this effect was mediated through the regulation of the expression of the cell cycle-related proteins, cyclin D1 and p21. This regulatory effect was exerted by restoring $\mathrm{H} 3 \mathrm{~K} 9 \mathrm{me} 3$ expression at the promoter regions of these genes. IOX1 may be thuse prove to be a potential therapeutic agent in the treatment of atherosclerosis.

\section{Acknowledgements}

This study was supported by grants from the National Natural Science Foundation of the People's Republic of China grants (nos. 81170195 and 81200156) and the Fundamental Research Funds for the Central Universities (no. 20120141120079). We would like to thank Professor Yanhong Tang, Professor Xi Wang, Dr Teng Wang and Dr Ping Hu from the Renmin Hospital of Wuhan University for their excellent technical assistance.

\section{References}

1. Libby P, Ridker PM and Hansson GK: Progress and challenges in translating the biology of atherosclerosis. Nature 473: 317-325, 2011.

2. Doran AC, Meller N and McNamara CA: Role of smooth muscle cells in the initiation and early progression of atherosclerosis. Arterioscler Thromb Vasc Biol 28: 812-819, 2008

3. King RW, Jackson PK and Kirschner MW: Mitosis in transition. Cell 79: 563-571, 1994.

4. Weiss D, Sorescu D and Taylor WR: Angiotensin II and atherosclerosis. Am J Cardiol 87: 25C-32C, 2001

5. Mehta PK and Griendling KK: Angiotensin II cell signaling: physiological and pathological effects in the cardiovascular system. Am J Physiol Cell Physiol 292: C82-C97, 2007.

6. Klein EA and Assoian RK: Transcriptional regulation of the cyclin D1 gene at a glance. J Cell Sci 121: 3853-3857, 2008.

7. Chen Z, Zang J, Kappler J, Hong X, Crawford F, Wang Q, Lan F, Jiang C, Whetstine J, Dai S, et al: Structural basis of the recognition of a methylated histone tail by JMJD2A. Proc Natl Acad Sci USA 104: 10818-10823, 2007.

8. Whetstine JR, Nottke A, Lan F, Huarte M, Smolikov S, Chen Z, Spooner E, Li E, Zhang G, Colaiacovo M and Shi Y: Reversal of histone lysine trimethylation by the JMJD2 family of histone demethylases. Cell 125: 467-481, 2006.

9. Zhang J, Chen J, Yang J, Xu C, Ding J, Yang J, Guo Q, Hu Q and Jiang H: Sodium ferulate inhibits neointimal hyperplasia in rat balloon injury model. PLoS One 9: e87561, 2014.

10. Shin S and Janknecht R: Activation of androgen receptor by histone demethylases JMJD2A and JMJD2D. Biochem Biophys Res Commun 359: 742-746, 2007.

11. Kogure M, Takawa M, Cho HS, Toyokawa G, Hayashi K, Tsunoda T, Kobayashi T, Daigo Y, Sugiyama M, Atomi Y, et al: Deregulation of the histone demethylase JMJD2A is involved in human carcinogenesis through regulation of the G(1)/S transition. Cancer Lett 336: 76-84, 2013.

12. Ding X, Pan H, Li J, Zhong Q, Chen X, Dry SM and Wang CY: Epigenetic activation of AP1 promotes squamous cell carcinoma metastasis. Sci Signal 6: ra28, 2013.

13. Berry WL, Shin S, Lightfoot SA and Janknecht R: Oncogenic features of the JMJD2A histone demethylase in breast cancer. Int J Oncol 41: 1701-1706, 2012.
14. Kauffman EC, Robinson BD, Downes MJ, Powell LG, Lee MM, Scherr DS, Gudas LJ and Mongan NP: Role of androgen receptor and associated lysine-demethylase coregulators, LSD1 and JMJD2A, in localized and advanced human bladder cancer. Mol Carcinog 50: 931-944, 2011.

15. Kim TD, Shin S, Berry WL, Oh S and Janknecht R: The JMJD2A demethylase regulates apoptosis and proliferation in colon cancer cells. J Cell Biochem 113: 1368-1376, 2012.

16. Gray SG, Iglesias AH, Lizcano F, Villanueva R, Camelo S, Jingu H, Teh BT, Koibuchi N, Chin WW, Kokkotou E and Dangond F: Functional characterization of JMJD2A, a histone deacetylase- and retinoblastoma-binding protein. J Biol Chem 280: 28507-28518, 2005.

17. Zhang D, Yoon HG and Wong J: JMJD2A is a novel N-CoRinteracting protein and is involved in repression of the human transcription factor achaete scute-like homologue 2 (ASCL2/Hash2). Mol Cell Biol 25: 6404-6414, 2005.

18. Villeneuve LM, Reddy MA, Lanting LL, Wang M, Meng L and Natarajan R: Epigenetic histone H3 lysine 9 methylation in metabolic memory and inflammatory phenotype of vascular smooth muscle cells in diabetes. Proc Natl Acad Sci USA 105: 9047-9052, 2008.

19. Weng $\mathrm{X}$, Cheng $\mathrm{X}$, Wu X, Xu H, Fang $\mathrm{M}$ and $\mathrm{Xu} \mathrm{Y}$ : Sin3B mediates collagen type I gene repression by interferon gamma in vascular smooth muscle cells. Biochem Biophys Res Commun 447: 263-270, 2014.

20. Lockman K, Taylor JM and Mack CP: The histone demethylase, Jmjd1a, interacts with the myocardin factors to regulate SMC differentiation marker gene expression. Circ Res 101: e115-e123, 2007.

21. Chen D, Liu J, Rui B, Gao M, Zhao N, Sun S, Bi A, Yang T, Guo Y, Yin Z and Luo L: GSTpi protects against angiotensin II-induced proliferation and migration of vascular smooth muscle cells by preventing signal transducer and activator of transcription 3 activation. Biochim Biophys Acta 1843: 454-463, 2014.

22. Kim JE and Choi HC: Losartan inhibits vascular smooth muscle cell proliferation through activation of AMP-activated protein kinase. Korean J Physiol Pharmacol 14: 299-304, 2010.

23. Molenaar JJ, Ebus ME, Koster J, van Sluis P, van Noesel CJ, Versteeg R and Caron HN: Cyclin D1 and CDK4 activity contribute to the undifferentiated phenotype in neuroblastoma. Cancer Res 68: 2599-2609, 2008.

24. Tsaousi A, Williams H, Lyon CA, Taylor V, Swain A, Johnson JL and George SJ: Wnt $4 / \beta$-catenin signaling induces VSMC proliferation and is associated with intimal thickening. Circ Res 108: 427-436, 2011

25. Musgrove EA: Cyclins: roles in mitogenic signaling and oncogenic transformation. Growth Factors 24: 13-19, 2006.

26. Lachner M, O'Carroll D, Rea S, Mechtler $\mathrm{K}$ and Jenuwein T: Methylation of histone $\mathrm{H} 3$ lysine 9 creates a binding site for HP1 proteins. Nature 410: 116-120, 2001.

27. Shen Q, Uray IP, Li Y, Krisko TI, Strecker TE, Kim HT and Brown PH: The AP-1 transcription factor regulates breast cancer cell growth via cyclins and E2F factors. Oncogene 27: 366-377, 2008.

28. Shaulian E and Karin M: AP-1 as a regulator of cell life and death. Nat Cell Biol 4: E131-E136, 2002.

29. Schiller R, Scozzafava G, Tumber A, Wickens JR, Bush JT, Rai G, Lejeune C, Choi H, Yeh TL, Chan MC, et al: A cell-permeable ester derivative of the JmjC histone demethylase inhibitor IOX1. ChemMedChem 9: 566-571, 2014.

30. King ON, Li XS, Sakurai M, Kawamura A, Rose NR, Ng SS, Quinn AM, Rai G, Mott BT, Beswick P, et al: Quantitative high-throughput screening identifies 8-hydroxyquinolines as cell-active histone demethylase inhibitors. PLoS One 5: e15535, 2010. 